This is the pre-peer reviewed version of the following article:

González-Monje P., Novio F., Ruiz-Molina D.. Covalent Grafting of Coordination Polymers on Surfaces: The Case of Hybrid Valence Tautomeric Interphases. Chemistry - A European J ournal, (2015). 21. : 10094 - . 10.1002/chem.201500671,

which has been published in final form at https://dx.doi.org/10.1002/chem.201500671. This article may be used for non-commercial purposes in accordance with Wiley Terms and Conditions for Use of Self-Archived Versions. 


\title{
Covalent Grafting of Coordination Polymers on Surfaces: the Case of Hybrid Valence Tautomeric Interphases
}

\author{
Pablo González-Monje ${ }^{[a]}$ Fernando Novio ${ }^{*[b]}$ and Daniel Ruiz-Molina ${ }^{\star[b]}$
}

\begin{abstract}
We have developed a novel approach for grafting coordination polymers, structured as nanoparticles bearing surface reactive carboxylic groups, to amino-functionalized surfaces through a simple carbodiimide-mediated coupling reaction. As a proof-of concept to validate our approach and on the quest for novel hybrid interphases with potential technological applications, we have used valence tautomeric nanoparticles exhibiting spin transition around room temperature. SEM and AFM characterization reveal that the nanoparticles were organized chiefly into a single monolayer while XPS measurements confirm that the nanoparticles retain a temperature-induced electronic redistribution upon surface anchorage. Our results represent an effective approach towards the challenging manufacturing of coordination polymers.
\end{abstract}

\section{Introduction}

Nanoscale coordination polymers, also known as coordination polymer particles (CPPs), exemplify a novel and innovative class of nanoscale solids created from the association of metal ions and multitopic organic ligands. ${ }^{[1]}$ The main interest sets in their unique physicochemical properties, high synthetic flexibility, rich chemistry and nanoscale dimensions, whose combination offers promising elements in diverse devices ranging from nanomedicine ${ }^{[2]}$ or (bio)sensors ${ }^{[3]}$ to molecular electronics. ${ }^{[4]}$ Whatever the application chosen, the perspective of implementation of these nanostructures requires in most of the cases the development of novel protocols to efficiently localize and organize them on a substrate (support) as the first step towards permanent hybrid materials for device fabrication and manufacturing. This is especially relevant for the case of switchable molecule-based magnetic materials where success will drive their use on new electronic or spintronic devices. Pioneering examples of prussian-blue like ${ }^{[5]}$ and spin-crossover nanoparticles $^{[6]}$ structured on surfaces by different means, mainly self-assembly or electrostatic interactions, have already been reported. Nonetheless, the synthetic methodology for organizing CPPs on surfaces is in its fledgling stage. Researchers are nowadays actively endeavoring to fully

[a] P. González-Monje,

Institut Catala de Nanociencia i Nanotecnologia (ICN2), Campus UAB, 08193 Bellaterra (Spain).

[b] F. Novio, D. Ruiz-Molina

Consejo Superior de Investigaciones Científicas (CSIC), Campus UAB, 08193 Bellaterra (Spain).

Institut Catala de Nanociencia i Nanotecnologia (ICN2), Campus UAB, 08193 Bellaterra (Spain). E-mail: druiz@cin2.es

Supporting information for this article is given via a link at the end of the document. understand the potential and limitations of these approaches, both for scientific reasons and for future technological applications. This is especially pertinent for the case of switchable valence tautomeric (VT) systems. VT represents a family of coordination complexes involving a reversible switching process between two electronic isomers with different spin ground states upon an intramolecular electron transfer takes place between a redox-active ligand and a metal ion. ${ }^{[7]}$ The different magnetic properties exhibited by both isomers, which are also accompanied by variations of the optical properties, can be easily tuned under the influence of various external parameters such as temperature, pressure or light irradiation opening up a broad range of possible applications in sensor, display and information technologies. ${ }^{[8,9]}$ However, VT complexes have been shown to exhibit a critical dependence on the local molecular environment with critical factors such as crystal packing, presence of solvate molecules and counterions, etc. ${ }^{[10]}$ This fact can restrict their proper surface integration without modifying the thermodynamics of the VT equilibrium.

Herein we report how the covalent grafting of VT-CPPs on surfaces represents a reliable approach to achieve this objective. For this we take advantage from the synthetic flexibility of amorphous CPPs (otherwise difficult to be achieved with crystalline frameworks) and the selection of the appropriate multitopic organic ligands bearing reactive chemical groups, in this specific case a carboxylic group. Those located at the surface of the nanoparticles are able to drive their 2-D covalent grafting on surfaces through a simple carbodiimide-mediated coupling reaction with an amino-functionalized surface and using the standard coupling reagents 1-ethyl-3-(3dimethylaminopropyl)carbodiimide (EDC) and Nhydroxysuccinimide (NHS). Moreover, these nanoparticles proved to be a highly robust platform, as it is reinforced by several hydrogen bonds within the polymeric network. ${ }^{[11]}$ This fact is expected to minimize the impact of the surface on the VT behavior of the nanoparticles upon surface attachment. A schematic representation of the synthetic approach used for the fabrication of the coordination polymer particles and their corresponding anchorage to surfaces through a simple carbodiimide-mediated coupling reaction are shown in Figure 1.

\section{Results and Discussion}

The coordination polymer of choice for these studies, [Co(3,5dhcSQ)(3,5-dhcCat)bix $]_{n}$, is obtained upon combination of the electroactive Co(3,5-dhcSQ)(3,5-dhcCat) units, where 3,5dhcSQ- and 3,5-dhcCat2- are respectively the semiquinonate radical and catecholate forms of 3,4-dihydroxycinnamic acid (dhc), with a bisimidazol 1,4-bis(imidazol-1-ylmethyl)benzene ligand (bix). ${ }^{[11]}$ 
a

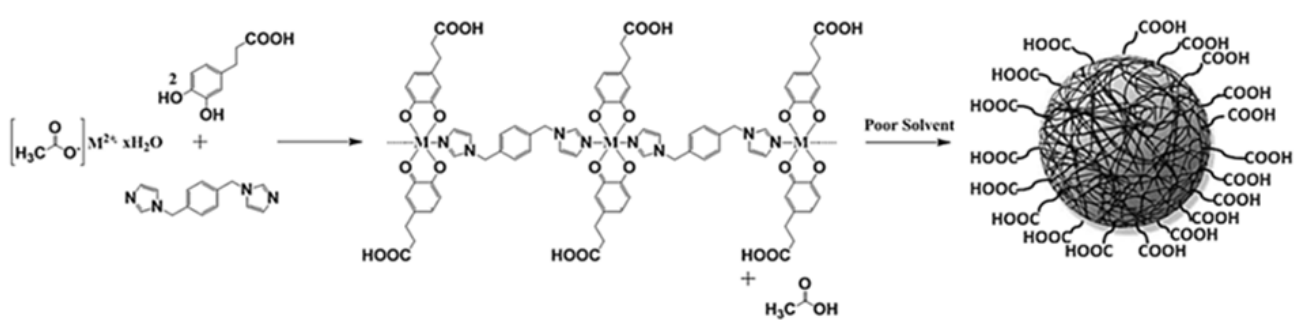

b

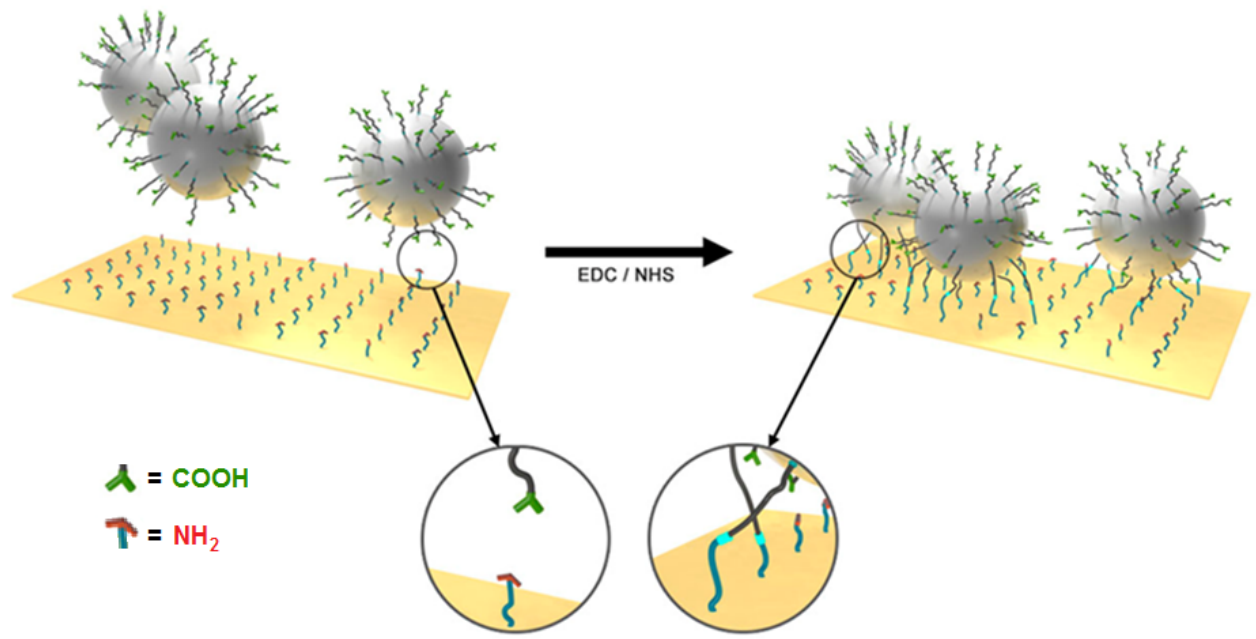

Figure 1. a) Schematic of the formation of polymeric chains for CPP-Co and structuration into a nanostructure by fast precipitation. b) Schematic of the attachment of carboxyl functionalized nanoparticles onto a gold surface previously covered with a monolayer of amino- terminated alkyl chains through carbodiimine mediated coupling reaction.

In a typical experiment, an aqueous solution of $\mathrm{Co}\left(\mathrm{CH}_{3} \mathrm{COO}\right)_{2} \cdot 2 \mathrm{H}_{2} \mathrm{O}$ was added to an ethanolic solution combining two co-ligands: dhc, used to introduce the carboxylic acid group on the structure, and bix, used as a counter ligand to induce polymerization. The precipitate was washed with ethanol, dried under vacuum and characterized (bulk characterization of CPP-Co nanoparticles is given in Experimental Section and Supporting Information, S1). The resulting product precipitates as nanoparticles with an average diameter of $128 \pm 19 \mathrm{~nm}$ on the basis of SEM, TEM and DLS measurements. X-ray diffraction (XRD) corroborated their amorphous structure. Magnetic susceptibility data of the bulk nanoparticles sample was measured in the 20-370 $\mathrm{K}$ temperature range. Even though the transition is not complete, the $\mu_{\text {eff }}$ value at $370 \mathrm{~K}$ is $4.4 \mu_{\mathrm{B}}$, close to the expected value for the $h s-\mathrm{Co}$ (II) isomer $\left(4.6 \mu_{\mathrm{B}}\right)$. On cooling, we observe an abrupt decrease of the $\mu_{\text {eff }}$ value down to a value of $3.6 \mu_{\mathrm{B}}$ at $310 \mathrm{~K}$ that is associated with a valence tautomeric interconversion of a fraction of molecules from the $h s-\mathrm{Co}(I I)$ to the $/ \mathrm{s}-\mathrm{Co}(\mathrm{III})$ isomer. Below $310 \mathrm{~K}$, the $\mu_{\text {eff }}$ value monotonically decreases down to a value of $2.6 \mu_{\mathrm{B}}$ at $40 \mathrm{~K}$, common for non-crystalline phases ${ }^{[12]}$ and tautomeric coordination polymers. ${ }^{[13]}$ The slow decrease has been attributed to: I) the gradual interconversion of the remaining high-spin fraction, II) spin-orbit coupling effects ${ }^{[14]}$ and/or III) the presence of an additional structural transition, as previously reported by Dei et al. ${ }^{[15]}$ This variable-temperature dependence is in agreement with the electronic equilibrium shown in Scheme 1. Raman spectra of the bulk sample at three different temperatures $293 \mathrm{~K}, 330 \mathrm{~K}, 373 \mathrm{~K}$ also confirmed the VT interconversion (see Supporting Information, S2).

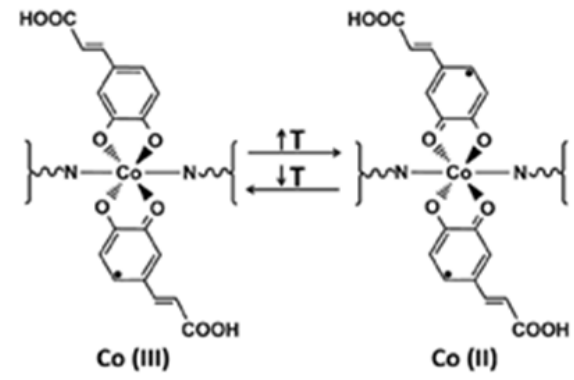

Scheme 1 
The first step towards their surface immobilization was the fabrication of an amino functionalized surface by self-assembly of 11-amino-1-undecanethiol (AUT) monolayer on a polycrystalline gold substrate. The gold substrate was previously washed in an ultrasonic bath for $15 \mathrm{~min}$ in acetonitrile, absolute ethanol and milli-Q water and dried with nitrogen gas. Afterwards, the substrate was then immersed in a $1 \mathrm{mM}$ ethanolic solution of AUT and left to soak overnight. The resulting $\mathrm{NH}_{2}$-self-assembly monolayer (SAM) was then heavily rinsed with absolute ethanol and finally, dried under an $\mathrm{N}_{2}$ stream. Surface immobilization was achieved by pre-incubating the nanoparticles in a buffer solution containing EDC in order to activate the carboxyl groups. Afterwards, the activated CPP-Co nanoparticles were organized over the functionalized gold surface following a two-step incubation process: 1) the functionalized gold surfaces were immersed in a buffer solution of CPP-Co nanoparticles at room temperature and 2) addition of NHS with overnight (ca. $18 \mathrm{~h}$ ) stirring ${ }^{[16,17]}$ and the following experimental conditions: $0.75 \mathrm{mg} / \mathrm{mL}$ CPP-Co, $0.25 \mathrm{mg} / \mathrm{mL}$ EDC, $0.15 \mathrm{mg} / \mathrm{mL} \mathrm{NHS}$, in sodium phosphate buffer (20nM), at pH 9.0. SEM and AFM images of the functionalized gold surface, after several washes with ethanol, clearly evidence that nearly 85$90 \%$ of the substrate was covered with the nanoparticles (see Figure 2a-b). Moreover, AFM images shown in Figure 2c reveal that the nanoparticles were organized chiefly into a single monolayer ranged in height from $75 \mathrm{~nm}$ to $225 \mathrm{~nm}$ (to ensure accurate size determination, only height measurements were used for data analysis, as lateral dimensions of nanoparticles measured by AFM topography are subject to tip convolution effects). These values range within those found in bulk for the same particles by SEM/TEM and DLS measurements. To demonstrate that fabrication of robust and functioning interfaces requires the covalent bonding, we also performed a control experiment in which the nanoparticles were simply incubated on an untreated gold surface (i.e. devoid of amino groups), using the same coupling conditions as above. Representative images of the control substrate after the cleaning process reveal a total absence of nanoparticles. Same results were obtained for a surface functionalized with amino groups in the presence of the nanoparticles but lacking the EDC and NHS coupling agents (see Supporting Information, S3).

Finally, the VT behavior of the nanoparticles upon surface immobilization was studied. For this, magnetization measurements were not feasible because of the small amount of material deposited, its low magnetic density and the observation of the magnetic transition around room temperature, which ruled out the use of surface confined scanning probe techniques. Micro-Raman ( $\mu R S)$ spectroscopy appears as an interesting alternative already used for the characterization of related spincrossover samples at the nanoscale, ${ }^{[18]}$. Unfortunately $\mu R S$ of the nanoparticle-covered gold substrate was not conclusive. While some changes on the most significant regions seemed to appear (see Supporting Information, S2), the signal turned out to be too small and therefore too noisy as to extract any conclusion. This is due most likely to the small amount of material present in the monolayer, possible interferences arising from the luminescence background of the sample or a combination of both.

a

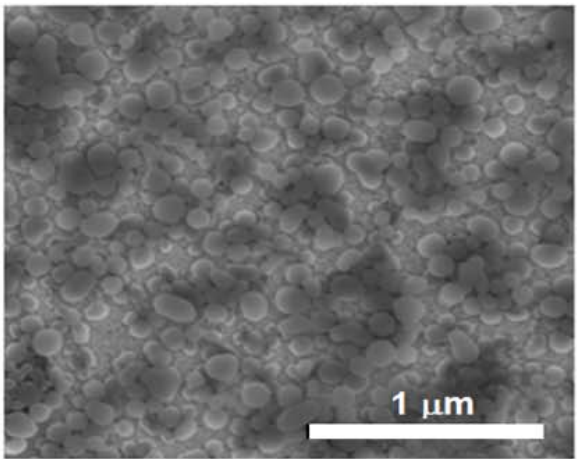

b

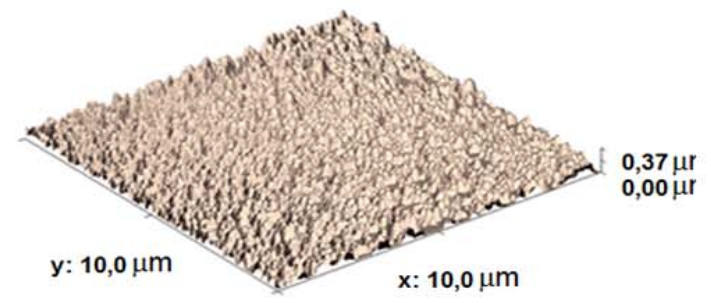

C

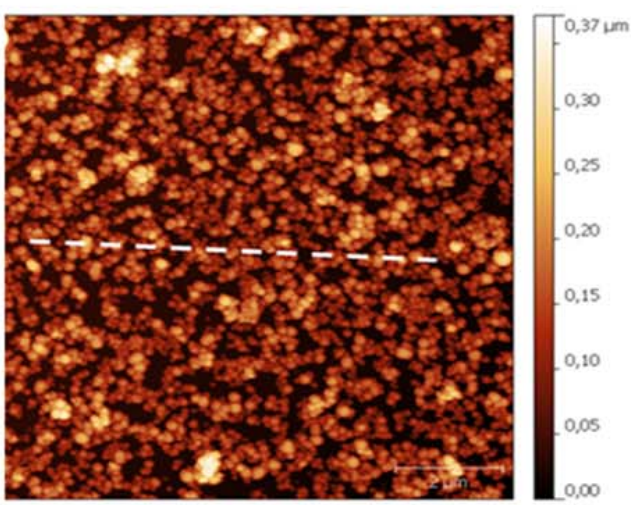

d

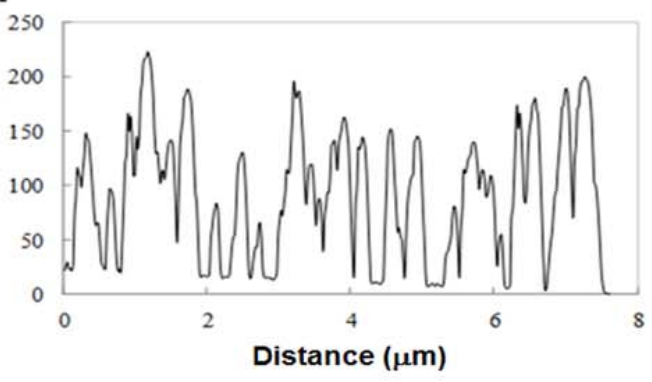

Figure 2. . a) SEM image of the CPP-Co chemically attached to the surface after condensation reaction; b-c), 3-D AFM topography image and topography image of the surface densely covered with CPP-Co, respectively; d) Height profile corresponding to white dashed line in (c). 
A temperature-induced electronic redistribution on CPP-Co nanoparticles upon surface immobilization was lastly confirmed by XPS measurements. This technique has already allowed the determination of charge distribution in different bulk VT complexes, both at fixed ${ }^{[19]}$ and variable temperatures, ${ }^{[20]}$ in addition to have sufficient sensitivity. Spectra were taken at three different temperatures: $80 \mathrm{~K}, 293 \mathrm{~K}$ and $373 \mathrm{~K}$. Best-fits and spin-orbit (SO) couplings within the Co2p region at the three different temperatures are shown in Figure 3. Analysis of the XPS data obtained showed signals in the $\mathrm{Co}_{2} 2 p_{3 / 2}$ region along with the corresponding $\mathrm{Co} 2 p_{1 / 2}$ spin-orbit coupled contributions weighted by the expected 2:1 ratio $(\mathrm{N} 1 \mathrm{~s}, \mathrm{C} 1 \mathrm{~s}$ and $\mathrm{O} 1 \mathrm{~s}$ contributions were discarded since they may be affected by spurious contaminations). The $\mathrm{Co} 2 p_{1 / 2}$ spectrums were then fitted to four components (along with the corresponding Co $2 p_{1 / 2}$ spin-orbit coupled contributions) following a previously reported procedure. ${ }^{[20]}$ At $80 \mathrm{~K}$, a main peak at $782.8 \mathrm{eV}$ and minor satellites at $786.0,789.2$ and $793.07 \mathrm{eV}$, with a $\Delta \mathrm{E}_{\mathrm{SO}}$ contribution of $15.2 \mathrm{eV}$ are obtained. These values are within the range of those previously described for a mixture of $I s-\mathrm{Co}$ (III) and $h s-\mathrm{Co}(\mathrm{II}) .{ }^{[20]} \quad$ An increase of the temperature reveals a related lineshape with a main peak shift to $782.4 \mathrm{eV}$ and satellites at $785.7,788.5$ and $792.0 \mathrm{eV}$ for the spectrum at $293 \mathrm{~K}$ and a main peak shift to 782.0 and satellites at $786.3,788.8$ and $792.0 \mathrm{eV}$ for the spectrum at $373 \mathrm{~K}$. The corresponding $\Delta \mathrm{E}_{\mathrm{SO}}$ contributions are 15.3 and $15.5 \mathrm{eV}$ for the $293 \mathrm{~K}$ and $373 \mathrm{~K}$, respectively.

The existence of a temperature-dependence in $\Delta \mathrm{E}_{\mathrm{SO}}$ is in agreement with a thermally driven electronic redistribution within the nanoparticles. Indeed, since SO depends on the cobalt redox state (being larger for the $h s-\mathrm{Co}$ (II) than for the $/ s-\mathrm{Co}$ (III) state) the increase of $\Delta \mathrm{E}_{\mathrm{SO}}$ on heating from $80 \mathrm{~K}$ to $373 \mathrm{~K}$ can be attributed to the reduction of the cobalt ion by an intramolecular electron transfer coming from the catechol-based ligand, i.e. with a VT process. However, comparison of the experimental $\Delta \mathrm{E}_{\mathrm{so}}$ values (ranging from $15.2 \mathrm{eV}$ and $15.5 \mathrm{eV}$.) with those previously reported for pure $I s-\mathrm{Co}$ (III) and $h s-\mathrm{Co}$ (II) complexes $\left(15.1 \mathrm{eV}\right.$ and $15.8 \mathrm{eV}$, respectively), ${ }^{[20]}$ reveal that over the whole temperature range studied no pure electronic states are found but rather the coexistence of both charge distributions, being the $/ s-\mathrm{Co}$ (III) predominant at low temperatures and the $h s-$ $\mathrm{Co}(\mathrm{II})$ electronic isomer at high temperature.

\section{Conclusions}

In summary, we have reported a new methodology for the chemically assisted assembly of nanoscale coordination polymers on substrates as 2-D monolayers through a stepwise formation and functionalization protocol. As far as we know, this is the first report for the controlled organization of CPPs on surfaces. First, we fabricated robust nanoparticles with enhanced thermal and colloidal stabilities by incorporation of terminal carboxyl group and the surface carboxyl groups can be subsequently reacted through well-known peptide couplings with an amino functionalized surface. As a proof-of concept to validate this approach, valence tautomeric CPP-Co nanoparticles were tested in view of their potential interest in future molecular electronic and storage devices. XPS experiments confirmed that the nanoparticles retain their temperature-induced electronic redistribution. The high flexibility of this strategy and its scalability to several different functional CPPs families will certainly drive novel research towards the fabrication of CPP-decorated surfaces with novel properties and applications. Main advantages are: I) our fabrication process is not altered by undesired molecule-substrate interactions since neither the morphology nor the functionality of the anchored nanoparticles are altered, II) this methodology can be extrapolated to a large variety of functionalities and reactive groups over several different substrates and devices and III) the rich physicochemical properties and synthetic flexibility of nanoscale coordination polymers ensures a broad range of applications for these new hybrid interfaces.

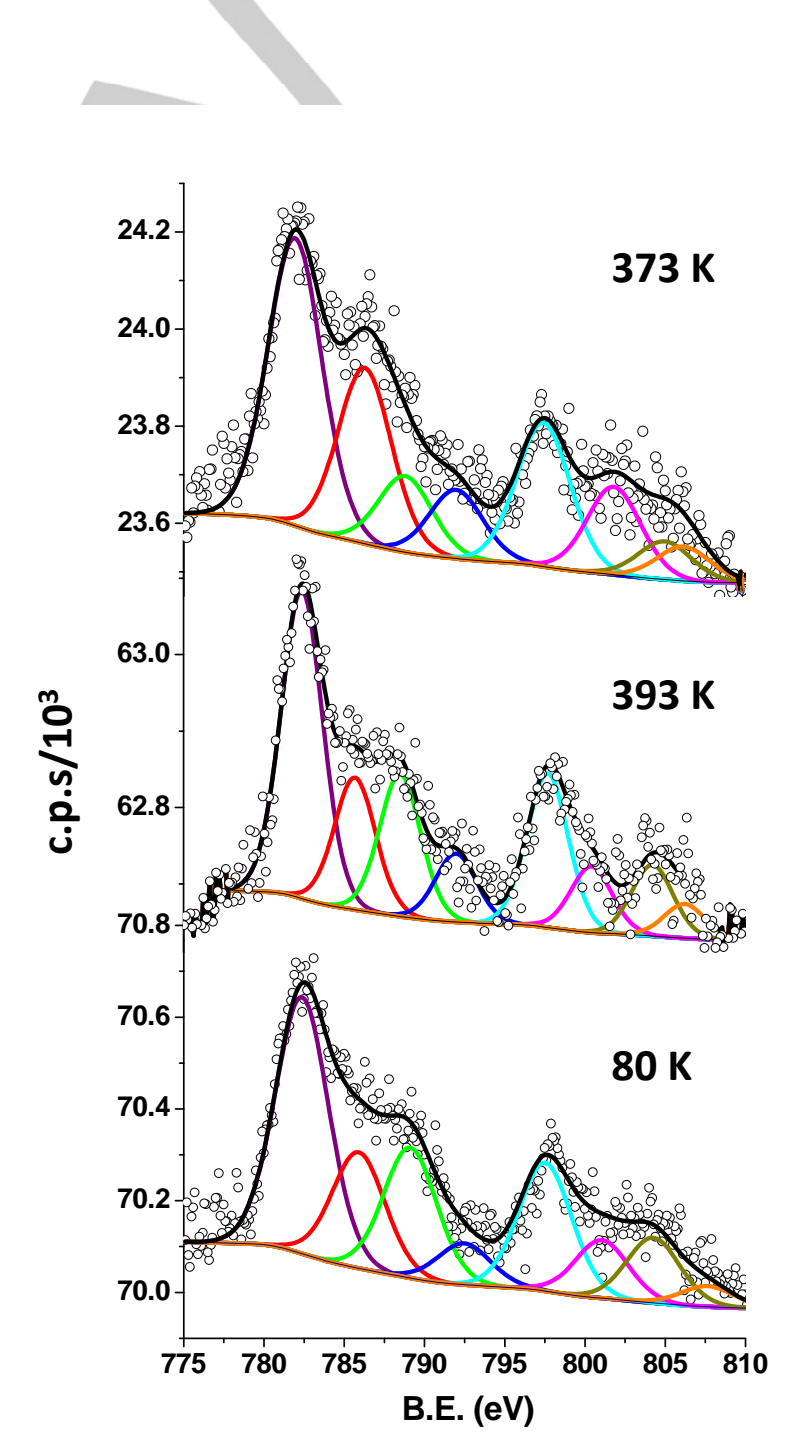

Figure 3. Variable-temperature Co2p XPS spectra of CPP-Co nanoparticles attached to a gold surface along with best-fit components. 


\section{Supporting Information}

Supporting Information is available from the Wiley Online Library or from the author.

\section{Experimental Section}

Solvents and starting materials were purchased from Sigma-Aldrich and used as received, without further purification, unless otherwise stated. 1,4-Bis(imidazol-1-ylmethyl)benzene (Bix) was synthesized according to a previously reported method. CPP-Co nanoparticles were synthesized according to a previously reported methodology. ${ }^{[11]}$

Fabrication of the 2-D nanoparticle arrays. CPP-Co NPs $(0.75 \mathrm{mg} / \mathrm{mL})$ were dispersed in a phosphate buffer solution at $\mathrm{pH} 9.0(20 \mathrm{mM})$, and the resulting solution/dispersion was treated with EDC $(0.25 \mathrm{mg} / \mathrm{mL})$, and then left overnight with magnetic stirring $(500 \mathrm{rpm})$. An AUTfunctionalized gold substrate was then immersed into the reaction solution, and the mixture was sonicated for 15 minutes, stirred magnetically ( $300 \mathrm{rpm}$ ) for 15 minutes and finally, left for 15 min to allow sedimentation of the nanoparticles. The previous procedure was repeated, and then the mixture was kept under magnetic stirring (300 $\mathrm{rpm}$ ) for $4.5 \mathrm{~h}$. Then, a solution of $0.15 \mathrm{mg} / \mathrm{mL} \mathrm{N}$-hydroxysuccinimide was added, and the gold substrate was kept overnight with magnetic stirring (500 to $700 \mathrm{rpm}$ ). Subsequently, the resulting CPP-Co functionalized gold substrate was rinsed thoroughly with milli-Q water and dried under an N2 stream. Several parameters were assessed, including the concentration of CPP-Co NPs $(0.75,0.5,0.05,0.025$ and $0.01 \mathrm{mg} / \mathrm{mL})$, the stoichiometry of NPS/EDC/NHS (10:5:3, 10:10:6, 15:5:3, 15:10:6), and the $\mathrm{pH}(7.0,9.0$ and 12.0); the best coupling results were obtained under the conditions described above. The other reaction conditions led to lower coupling rates, as indicated by less surface-bound CPPs, less surface area covered, and higher concentrations of aggregated nanoparticles. Additionally, several different incubation procedures were tested: i) using only ultrasounds, and/or stirring, and/or sedimentation, or a combination of them in order to carry out the immobilization of the CPP-Co to the AUT-functionalized substrate, ii) varying the time of addition of NHS. The stoichiometric ratio of EDC to NHS was kept constant in all the experiments.

Equipment. Scanning electron microscopy (SEM): SEM measurements were performed with a DITACHI S-570 operating at $15 \mathrm{kV}$. The samples were prepared by drop casting of the corresponding dispersion on aluminium tape followed by evaporation of the solvent under room conditions. Before analysis the samples were metalized with a thin layer of gold, using a sputter coater (Emitech K550). SEM images for immobilization of CPPs onto solid supports were taken with FEI Magellan 400L XHR operating at $3.0 \mathrm{kV}$ and $10-5 \mathrm{~Pa}$ of vacuum in the chamber. Transmission electron microscopy (TEM): TEM images were acquired with a Jeol JEM-1400 microscope operating at $120 \mathrm{kV}$. The samples were prepared by casting a drop of the corresponding sample dispersion on a holey carbon copper grid, and then evaporating off the solvent under room temperature. Dynamic light scattering (DLS): Size distribution and surface charge of the nanoparticles were measured by DLS, using the Zetasizer Nano 3600 instrument (Malvern Instruments, UK), whose size range limit is $0.6 \mathrm{~nm}$ to $6 \mu \mathrm{m}$. Note: the diameter measured by DLS is the hydrodynamic diameter. The samples comprised aqueous dispersions of the nanoparticles in distilled water or in buffer. All samples were diluted to obtain an adequate nanoparticle concentration. The data reported are mean values for each sample, which were measured in quadruplicate. Powder X-ray diffractometry (XRD): Powder XRD spectra were recorded at room temperature on a high-resolution texture diffractometer (PANalytical X'Pert PRO MRD) equipped with a Co-Ka radiation source $(I=1.7903 \AA)$ and operating in reflection mode. The solid samples were placed in an amorphous silicon oxide flat plate and measured directly. Atomic force microscopy (AFM): Dynamic non-contact mode AFM images were acquired on an Agilent 5500 AFM/SPM microscope. Non-contact high-resonance frequency (NCHR) tips with reflex coating were used-namely, PPP-NCHR silicon point probes (spring constant: ca. $42 \mathrm{~N} / \mathrm{m}$; resonant frequency: ca. $330 \mathrm{kHz}$; from Nanosensors). The AFM images were processed and rendered using Gwyddion data analysis software. Micro-Raman microscopy: Raman spectra were acquired at different temperatures using a Dilor triplemate spectrograph (1800 1/mm grating, $100 \mathrm{~lm}$ entrance slit, $1 \mathrm{~cm}-1$ spectral resolution) coupled to a Princeton Instruments CCD detector. The 647.1$\mathrm{nm}$ line of a $\mathrm{Kr}+$ laser (Coherent RadiationInnova) was used as an excitation source with laser power output of $10 \mathrm{~mW}$. The laser beam has been focused on a spot of about $3 \mu \mathrm{m}$ in diameter and the Raman signal has been collected in a backscattering geometry. The establishment of the spin-equilibrium was inferred from the stabilisation of the Raman signal intensity. The same performance was carried out for the sample in bulk and the nanoparticles monolayer attached to the gold surface. Infrared (IR) spectrophotometry: The IR spectra have been recorded using a Tensor 27 (Bruker) spectrophotometer equipped with a single reflexion diamond window ATR accessory (MKII Golden Gate - Specac). $X$-Ray Photoelectron Spectroscopy: XPS experiments were performed in a Phoibos 150 analyzer (SPECS $\mathrm{GmbH}$, Berlin,Germany) in ultra-high vacuum conditions (base pressure $1 \cdot 10^{-10} \mathrm{mbar}$ ). A monochromatic Al $\mathrm{Ka}$ source (1486.7 eV) operating at $400 \mathrm{~W}$ was used. Wide scans were acquired at analyzer pass energy of $50 \mathrm{eV}$, while high resolution narrow scans were performed at constant pass energy of $20 \mathrm{eV}$ and steps of 0.1 $\mathrm{eV}$. The photoelectrons were detected at a takeoff angle $\Phi=0^{\circ}$ with respect to the surface normal. The spectra were obtained at room temperature. The binding energy $(\mathrm{BE})$ scale was internally referenced to the $C 1$ s peak (BE for $C-C=284.8 \mathrm{eV}$ )

\section{Acknowledgements}

F. N. thanks the Ministerio de Ciencia e Innovación (MICINN) for a postdoctoral JdC fellowship. This work was supported by project MAT2012-38318-C03-02 from the Spanish Government and by FEDER funds. ICN2 acknowledges support from the Severo Ochoa Program (MINECO, Grant SEV-2013-0295) Authors also thank MP1202 Cost Action and Dr. G. Molnar and Prof. A. Bousseksou for Raman measurements and very helpful discussions. We also thank G. Sautier for XPS measurements and helpful disscusion.

Keywords: coordination polymer, nanoparticles, spin transition, 2-D assembly, surface assembly, valence tautomerism

[1] a) M. Y. Masoomi, A. Morsali, RSC Adv., 2013, 3, 19191; b) F. Novio, J. Simmchen, N. Vázquez-Mera, L. Amorín-Ferré, D. Ruiz-Molina, Coord. Chem. Rev. 2013, 257, 2839; c) W. Lin, W. J. Rieter, K. M. L. Taylor Angew. Chem. Int. Ed., 2009, 48, 650; d) A. M. Spokoyny, D. Kim, A. Sumrein, C. A. Mirkin, Chem. Soc. Rev., 2009, 38, 1218

[2] a) S. Bhattacharjeea, S. Bhattacharya, Chem. Commun., 2014, 50, 11690; b) S. Xu, J. Liu, D. Li, L. Wang, J. Guo, Ch. Wang, Ch. Chen, Biomaterials 2014, 35, 1676; c) P. Fei Gao, L. L. Zheng, L. J. Liang, X. X. Yang, Y. F. Li, Ch. Z. Huang, J. Mater. Chem. B, 2013, 1, 3202; d) L. Xing, Y. Cao, S. Che, Chem. Commun. 2012, 48, 5995.e) R. C. Huxford, K. E. deKrafft, W. S. Boyle, D. Liu, W. Lin, Chem. Sci., 2012, 3, 198; f) I. Imaz, M. Rubio-Martínez, L. García-Fernández, F. García, D. Ruiz-Molina, J. Hernando, V. Puntes, D. Maspoch, Chem. Commun., 
2010, 46, 4737; g) R. Nishiyabu, C. Aimé, R. Gondo, T. Noguchi, N Kimizuka, Angew. Chem., Int. Ed., 2009, 48, 9465.

[3] a) G. Paul, Y. Prado, N. Dia, E. Rivière, S. Laurent, M. Roch, L. Vander Elst, R. N. Muller, L. Sancey, P. Perriat, O. Tillement, T. Mallah, L. Catala Chem. Commun., 2014, 50, 6740; b) H. Tan, Ch. Ma, Y. Song F. Xu, Sh. Chen, L. Wang Biosensors and Bioelectronics 2013, 50, 447; c) F. Leng, X. J. Zhao, J. Wang, Y. F. Li Talanta 2013, 107, 396; d) H. Tan, L. Zhang, Ch. Ma, Y. Song, F. Xu, Sh. Chen, L. Wang ACS Appl. Mater. Interfaces 2013, 5, 1179 .

[4] a) I. A. Gural'skiy, C. M. Quintero, G. Molnár, I. O. Fritsky, L. Salmon, A. Bousseksou, Chem. Eur. J., 2012, 18, 9946; b) F. Prins, M. MonrabalCapilla, E. A. Osorio, E. Coronado, H. S. J. van der Zant, Adv. Mater. 2011, 23, 1545; c) I. Boldog, A. Gaspar, V. Martínez, P. Pardo-lbañez, V. Ksenofontov, A. Bhattacharjee, P. Gütlich, J. A. Real, Angew. Chem. Int. Ed., 2008, 47, 6433.

[5] a) E. Coronado , A. Forment-Aliaga, E. Pinilla-Cienfuegos, S. Tatay, L. Catala , J. A. Plaza Adv. Funct. Mater. 2012, 22, 3625; b) M. ClementeLeón, E. Coronado, Á. López-Muñoz, Diego Repetto, L. Catala, T. Mallah Langmuir 2012, 28, 4525-4533; c) F. Volatron, D. Heurtaux, L. Catala, C. Mathonière, A. Gloter, O. Stéphan, D. Repetto, M. ClementeLeón; E. Coronado, T. Mallah Chem. Commun., 2011, 47, 1985; d) M. Clemente-León, E. Coronado,Á. López-Muñoz, D. Repetto, Ch. Mingotaud, D. Brinzei, L. Catala, T. Mallah Chem. Mater. 2008, 20, 4642; e) A Ghirri, A. Candini, M. Evangelisti, G. C. Gazzadi, F. Volatron, B. Fleury, L. Catala, Ch. David, T. Mallah, M. Affronte Small 2008, 4, 2240.

[6] a) D. Gentili, F. Valle, C. Albonetti, F. Liscio, M. Cavallini; Acc. Chem. Res., 2014, 47, pp 2692; b) A. Bousseksou, G. Molnár, L. Salmon, W. Nicolazzi, Chem. Soc. Rev., 2011, 40, 3313; c) F. Prins, M. MonrabalCapilla, E. A. Osorio, E. Coronado,H. S. J. van der Zant Adv. Mat. 2011, 23, 1545; d) M. Cavallini, I. Bergenti, S. Milita, J. Crispin Kengne, D. Gentili, G. Ruani, I. Salitros , V. Meded, M. Ruben, Langmuir, 2011, 27, 4076.

[7] a) T. Tezgerevska; K. G. Alley; C. Boskovic Coord. Chem. Rev. 2014, 268, 23. b) E. Evangelio; D. Ruiz-Molina, Eur. J. Inorg. Chem. 2005 2957.

[8 ] a) A.Dei, D. Gatteschi, Angew. Chem. Int. Ed. Engl. 2011, 50, 11852 ; b) O. Sato, J. Tao, Y. -Z. Zhang, Angew. Chem. Int. Ed. Engl. 2007, 46, 2152 .
[9] a) E. Ruiz, Phys. Chem. Chem. Phys. 2014, 16, 1, 14; b) G. Molnár, L. Salmon, W. Nicolazzi, F. Terki, A. Bousseksou, J. Mat. Chem. C, 2014 2, 1360; c) A. Rotaru, J. Dugay, R. P. Tan, I. A. Guralskiy, L. Salmon, Ph. Demont, J. Carrey, G. Molnár, M. Respaud, A. Bousseksou, Adv. Mat. 2013, 25, 1745; d) D. Chiruta, J. Linares, M. Dimian, Y. Alayli, Y. Garcia, Eur. J. Inorg. Chem. 2013, 29, 5086-5093; e) F. Prins, M. Monrabal-Capilla, E. A. Osorio, E. Coronado, H. S. J. van der Zant, Adv. Mat. 2011, 23, 1545

[10] a) E. Evangelio, D. Ruiz-Molina, C. R. Chimie 2008, 11, 1137-1154; b) E. Evangelio, Cl. Rodriguez-Blanco, Y. Coppel, D. N. Hendrickson, J. P. Sutter, J. Campo, D. Ruiz-Molina, Solid State Sciences 2009, 11, 793800.

[11] a) F. Novio, D. Ruiz-Molina, RSC Adv., 2014, 4, 15293; b) F. Novio, J. Lorenzo, F. Nador, K. Wnuk, D. Ruiz-Molina, Chem. Eur. J. 2014, 20 , 15443.

[12] S. H. Bodnar, A. Caneschi, A. Dei, D. A. Shultz, L. Sorace, Chem. Commun. 2001, 2150.

[13] I. Imaz, D. Maspoch, Cl. Rodríguez-Blanco, J. Manuel Pérez-Falcón, J. Campo, D. Ruiz-Molina Angew. Chem. Int. Ed. Engl. 2008, 120, 1883.

[14] F. Lloret, M. Julve, J. Cano, R. Ruiz-García and E. Pardo, Inorg. Chim. Acta 2008, 361, 3432.

[15] a) M. Affronte, A. Beni, A. Dei, L. Sorace, Dalton Trans., 2007, 5253; b) A. Beni, A. Dei, D. A. Shultz, L. Sorace, Chem. Phys. Lett. 2006, 428, 400.

[16] A. Bousquet, E. Ibarboure, C. Labrugere, E. Papon, J. RodríguezHernández, Langmuir 2007, 23, 6879.

[17] A. Shavel, N. Gaponik, A. Eychmüller, ChemPhysChem 2005, 6, 449

[18] a) G. Molnár, A. Bousseksou, A. Zwick, J. J. McGarvey, Chem Phys. Lett. 2003, 367, 593; b) S. Bedoui, G. Molnár, S. Bonnet, C. Quintero H. J. Shepherd, W. Nicolazzi, L Salmon, A. Bousseksou, Chem. Phys. Lett. 2010, 499, 94; c) J. A. Wolny, R. Diller, V. Schünemann, Eur. J. Inorg. Chem. 2012, 2635.

[19] H. Ohtsu, K. Tanaka, Chem. Eur. J. 2005, 11, 3420

[20] G. Poneti,M. Mannini, Br. Cortigiani, L. Poggini, L. Sorace, E. Otero, Ph. Sainctavit, R. Sessoli, A. Dei Inorg. Chem. 2013, 52, 11798. 


\section{FULL PAPER}

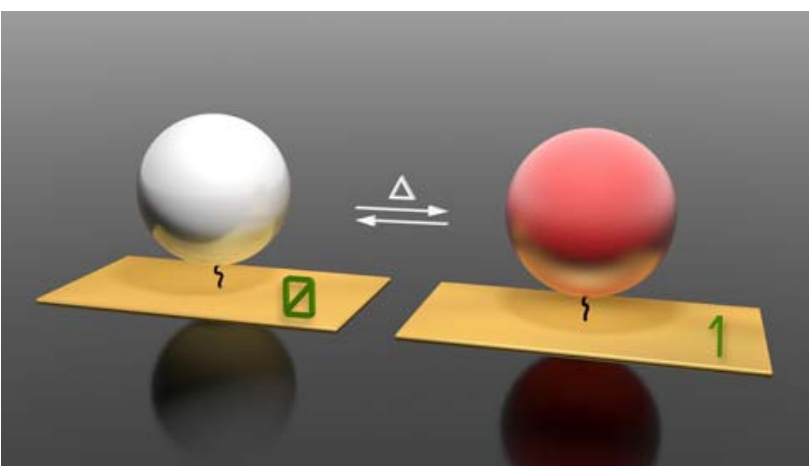

P. González-Monje, F. Novio* and D. Ruiz-Molina *

Page No. - Page No.

Covalent Grafting of Coordination Polymers at Surfaces: the Case of Hybrid Valence Tautomeric Interphases 